\title{
L'auto-confrontation : outil d'observation du développement de l'agir d'enseignants novices
}

\section{Véronique Laurens}

\section{(2) OpenEdition}

Journals

Édition électronique

URL : http://journals.openedition.org/rdlc/773

DOI : $10.4000 /$ rdlc. 773

ISSN : 1958-5772

Éditeur

ACEDLE

Référence électronique

Véronique Laurens, «L'auto-confrontation : outil d'observation du développement de l'agir

d'enseignants novices », Recherches en didactique des lanques et des cultures [En ligne], 12-2 | 2015,

mis en ligne le 20 novembre 2015, consulté le 19 avril 2019. URL : http://journals.openedition.org/ rdlc/773 ; DOI : 10.4000/rdlc.773

Ce document a été généré automatiquement le 19 avril 2019

\section{$(1) \Theta \Theta$}

Recherches en didactique des langues et des cultures is licensed under a Creative Commons AttributionNonCommercial-NoDerivatives 4.0 International License 


\title{
L'auto-confrontation : outil d'observation du développement de l'agir d'enseignants novices
}

\author{
Véronique Laurens
}

\section{Introduction}

1 Le recours à l'auto-confrontation pour faire produire des verbalisations sur leur agir par des enseignants est largement répandu dans les recherches sur la pensée enseignante et les pratiques effectives des enseignants en sciences de l'éducation et en didactique des langues (cf. par exemple, Tochon, 2000 ; Clot \& Faïta, 2000 ; Borg, 2006 ; Plazaola Giger \& Stroumza, 2007; Bigot \& Cadet, 2011; Cicurel, 2011; Rivière \& Cadet, 2011). Cette technique constitue une voie d'accès possible à la dimension cachée des interactions entre apprenants et enseignant. Par dimension cachée, nous entendons ce qui sous-tend la fabrique de l'action enseignante, c'est-à-dire l'ensemble des représentations, croyances, savoirs et modèles (Cambra Giné, 2003; Garcia-Debanc, 2008; Causa, 2012) constituant le répertoire didactique de l'enseignant (Cicurel, 2002; Causa, 2012) et mobilisé par celui-ci pour décider et agir au fil des interactions avec les apprenants.

La technique de l'auto-confrontation simple a été utilisée dans le cadre d'une recherche consacrée au développement de l'agir d'enseignants novices, à partir des liens qui peuvent se tisser entre les contenus méthodologiques travaillés en formation d'enseignants de français langue étrangère (FLE) et le passage à la pratique lors de premières prises en main de cours (Laurens, 2013). Cette recherche a consisté à observer comment des enseignants novices mobilisent les savoirs d'ingénierie travaillés en formation au niveau de la conception et de l'animation d'unités didactiques. À partir de cette recherche, la présente contribution vise à cerner en quoi l'analyse de verbalisations recueillies dans le cadre d'entretiens d'auto-confrontation (EAC) permet de cerner le 
développement de l'agir d'enseignants novices, et leur appropriation en marche de savoirs d'ingénierie travaillés en formation.

Dans un premier temps, cet article reviendra sur la notion d'agir enseignant, ce qui permettra ensuite de préciser le recours à l'EAC d'un point de vue méthodologique dans le cadre de la recherche menée. Dans un deuxième temps, nous illustrerons notre propos par l'analyse des commentaires recueillis auprès d'une enseignante novice observée, lors de deux EAC. Une vue d'ensemble des verbalisations sera d'abord présentée au moyen d'un classement en fonction de critères liés aux trois pôles du triangle didactique (commentaires liés à l'enseignant, à l'apprenant ou aux objets de savoirs). Puis, l'analyse se concentrera sur les verbalisations à dimension méthodologique afin de cerner en quoi ils sont révélateurs de l'agir en construction de l'enseignante observée, dans le lien avec les savoirs d'ingénierie abordés en formation.

\section{Observer le développement de l'agir enseignant}

\section{L'agir enseignant : situer l'enseignant comme auteur d'actions}

La notion d'agir enseignant a été développée par Cicurel $(2005,2007,2011)$ dans le cadre de l'orientation actionnelle des recherches sur les discours de la classe de langue. À partir du milieu des années 2000, l'analyse des interactions didactiques connaît une inflexion vers l'action, en didactique des langues et en sciences de l'éducation (cf. Cicurel \& Bigot, 2005 ; Filliettaz \& Schubauer-Leoni, 2008). Cette orientation met l'accent sur les actions telles qu'elles émergent au fil des interactions : "Travailler en classe implique non seulement l'émergence de discours, mais aussi d'actions produites en vue de l'acquisition de savoirs et savoirfaire langagiers." (Rivière, 2005 : 96). Le regard porté sur l'enseignant évolue, il s'agit de l'envisager non seulement comme "l'interactant d'une classe, mais aussi comme l'auteur d'actions" (Cicurel, $2005: 24)$.

5 Les actions de l'enseignant n'étant qu'en partie interprétables à partir des interactions, on essaie d'en cerner les intentions sous-jacentes. Ceci peut se faire en élargissant les analyses aux verbalisations de l'enseignant sur ses actions, ce qui permet de tenter d'avoir accès à la dimension cachée de l'agir enseignant (la genèse de l'action, les motifs, l'intentionnalité, la prise de décisions) (Cicurel, $2011: 240$ ). L'agir enseignant est ainsi vu comme constitué "[...] par la somme des actions, des intentions, des émotions et des projets de l'enseignant" (ibid. : 239).

6 L'appui sur la notion d'agir en didactique des langues représente un intérêt certain pour notre propos car cela permet, comme le souligne Cicurel (2005), de reconsidérer la perspective praxéologique de la discipline, notamment dans l'articulation avec la formation. On peut ainsi observer le lien entre formation et agir à partir des pratiques effectives repérées au fil des interactions et éclairées par les verbalisations des enseignants.

\section{L'agir enseignant : entre inscription dans le collectif d'une profession et actualisation de son action individuelle en situation}

7 L'autre intérêt de la notion d'agir, dans le cadre de notre recherche, consiste dans le fait qu'elle peut être distinguée en "activité" dans son entour collectif et en "action" dans sa 
dimension individuelle. C'est ce que pose Bronckart dans le cadre de l'interactionnisme socio-discursif (1996). C'est à partir de l'activité que l'action est construite par l'individu :

[...] les formes d'action ne sont pas d'abord des productions sui generis de la pensée ou de la conscience des actants individuels ; elles sont des produits de mécanismes interactifs complexes, auxquels participent certes ces actants mais qui ne peuvent se déployer que dans le cadre plus ou moins contraignant d'activités et de préconstruits collectifs toujours historiquement déjà-là. (Bronckart, 2010 : 86)

8 Dans la lignée de Bronckart, Filliettaz (2005) précise la distinction entre activité et action en s'appuyant sur la notion de typification développée par Schütz en sociologie phénoménologique (1987). La typification renvoie au fait que les actions accomplies par un individu sont ancrées dans ses expériences passées et sont en partie façonnées par elles. De plus, les actions d'un individu étant déployées socialement, elles ont des points communs avec celles d'autres individus. Ainsi, la conception typifiante de l'agir chez Schütz consiste à poser que les individus "disposent de ressources préalables pour agir" qui ne sont pas "propres aux individus" mais qui relèvent $d$ '"un processus fondamentalement collectif et culturel" (Filliettaz, 2005 : 24). À partir de la notion de typification, Filliettaz propose donc de distinguer les activités typifiées à dimension collective d'un côté et les actions situées individuelles de l'autre.

9 Si l'on considère le ou les premiers cours assurés par un enseignant novice (de FLE), son agir peut être vu en termes d'actions au sens où l'on va s'attacher à décrire ses manières de faire individuelles singulières et spécifiques à la situation d'enseignement/ apprentissage, et, il peut être considéré également dans la perspective des types d'activités didactiques/pédagogiques auxquelles il renvoie, dans un rapport dialectique entre action et activité formant l'agir :

[...] les actions situées s'adossent à des activités disponibles au plan sociohistorique; et celles-ci se trouvent en permanence révélées et réalimentées par les actions effectives et les évaluations qui en sont faites dans les situations dans lesquelles elles prennent place. (ibid. : 25)

La distinction activité/action de l'agir fait écho pour nous à celles de genre et de style professionnels développées en psychologie du travail (Clot, 1999 ; Clot \& Faïta, 2000). Clot ne s'appuie pas sur la notion d'agir et sa distinction en activité et action, mais il considère l'activité du sujet au travail dans ses dimensions individuelle et collective, afin de saisir la place et le rôle de chacune de ces dimensions dans le déploiement de l'activité et au niveau du développement du sujet au travail (1999: 9). Pour ce faire, Clot transpose les notions de genre et de style empruntées à Bakhtine (1984) dans le domaine de l'ergonomie du travail : l'activité du sujet se déploie selon son style propre dans le cadre d'un genre d'activité. Ainsi, le genre professionnel, tel que celui de l'enseignement des langues, peut être considéré comme une sorte de "[p]ré-fabriqué, stock de 'mises en actes' et de 'mises en mots' prêts à parler, c'est aussi une mémoire pour pré-dire" (Clot, 1999 : 42). Le sujet enseignant façonne dans son action son style individuel de l'activité et pour ce faire, il s'appuie sur les activités du genre professionnel auquel il se rattache. Ce faisant, le genre professionnel est actualisé dans l'action stylisée du sujet, c'est-à-dire renouvelé par elle (Clot \& Faïta, $2000: 15$ ).

11 Dans le cadre de notre recherche, on conçoit l'agir enseignant comme se déployant entre des activités typifiées "déjà-là" liées à un genre professionnel et à des actions situées caractérisant un style professionnel. Les savoirs d'ingénierie abordés en formation (principes didactiques/méthodologiques et techniques méthodiques/pédagogiques) se situent du côté du collectif, de l'activité et du genre professionnel, en tant qu'ensemble 
hérité sédimenté de plusieurs configurations didactiques, d'une part, présentant un ensemble d'activités typifiées appartenant au genre professionnel de l'enseignement des langues, d'autre part. Observer le développement de l'agir d'enseignants novices, en lien avec les savoirs d'ingénierie travaillés en formation, consiste donc à repérer les actions situées des enseignants (leurs pratiques effectives) actualisant (ou non) les savoirs d'ingénierie selon leur style professionnel en construction.

\section{Construire une démarche de recherche avec les EAC}

\section{Analyser les verbalisations des enseignants sur leur agir}

\section{La technique de l'auto-confrontation}

vise à donner au sujet un accès à des traces de son action notamment par la voie du filmage. Le protocole de ce type de recueil de données demande [...] au sujet de produire un discours [sur son agir] face aux séquences filmées. (Cicurel, $2011: 250$ )

Les verbalisations récoltées dans le cadre de ces EAC permettent d'entrevoir l'épaisseur du développement de l'agir, en faisant émerger des phénomènes qui n'apparaissent pas à l'observation des interactions, comme par exemple, l'activité de planification, la gestion du temps, les émotions, les obstacles, etc. (Cicurel \& Rivière, 2008 : 264-266).

Dans l'analyse menée ici, nous avons procédé, sur un plan macro, par l'analyse de contenus des commentaires produits en fonction des trois pôles du triangle didactique auxquels ils se rapportent: l'enseignant, les apprenants et les objets de savoirs. Ce découpage de la teneur des commentaires vise à cibler, sur le plan micro, les propos des enseignants relatifs aux savoirs d'ingénierie qu'ils mobilisent, c'est-à-dire à leurs manières de faire et à leurs convictions méthodologiques.

\section{Recueil et traitement des données}

\section{Contexte du recueil des données}

Dans le cadre de la recherche menée, nous avons pu suivre les premières prises en main de cours d'Alice, enseignante stagiaire, étudiante en master de didactique des langues à l'Université Paris Descartes ${ }^{1}$, à l'occasion de deux cours (codés ALICE 1 et ALICE 2) qu'elle a animés avec des étudiants chinois de l'université ${ }^{2}$ lors du stage d'enseignement s'étant déroulé au premier semestre de sa deuxième année de master (2009-2010). Les deux cours d'Alice ont été filmés et transcrits, et ont ensuite donné lieu à la réalisation de deux entretiens d'auto-confrontation. Nous détaillons ci-après dans quatre tableaux les informations générales sur les deux cours, les objets enseignés et les activités mises en œuvre.

Tableau 1 - Informations générales sur les deux cours.

\begin{tabular}{|l|l|l|}
\hline & ALICE 1 & ALICE 2 \\
\hline Date des cours & 16.10 .09 & 8.01 .10 \\
\hline Durée des cours & $51^{\prime} 01^{\prime \prime}$ & $50^{\prime} 17^{\prime \prime}$ \\
\hline
\end{tabular}




\begin{tabular}{|l|l|l|}
\hline Tours de paroles identifiés & 514 & 422 \\
\hline Date de l'EAC & 16.03 .10 & $9.4 .10 \& 16.4 .10$ \\
\hline Durée de l'EAC & $2 \mathrm{~h}$ & $1 \mathrm{~h} 30+1 \mathrm{~h} 30$ \\
\hline Nombre de commentaires produits & 17 & 30 \\
\hline
\end{tabular}

Tableau 2 - Présentation des objets enseignés dans les cours ALICE 1 et ALICE 2.

\begin{tabular}{|c|c|c|c|}
\hline & & ALICE 1 & ALICE 2 \\
\hline \multicolumn{2}{|l|}{ Tâche } & $\begin{array}{l}\text { Déguster un vin (donner ses } \\
\text { caractéristiques et sa région) }\end{array}$ & $\begin{array}{l}\text { Découvrir l'exposition De Byzance à } \\
\text { Istanbul (et s'y rendre) }\end{array}$ \\
\hline \multirow{3}{*}{ Objectifs } & Pragmatique & Décrire un vin & $\begin{array}{l}\text { Situer les dates de l'histoire de la ville } \\
\text { d'Istanbul }\end{array}$ \\
\hline & $\begin{array}{l}\text { Socio- } \\
\text { culturel }\end{array}$ & $\begin{array}{l}\text { Les vins de France et leurs } \\
\text { régions, la dégustation des } \\
\text { vins }\end{array}$ & $\begin{array}{l}\text { La ville d'Istanbul dans l'histoire, les } \\
\text { époques historiques d'Europe }\end{array}$ \\
\hline & Linguistique & $\begin{array}{l}\text { Adjectifs qualificatifs et noms } \\
\text { spécifiques (œnologie) } \\
\text { Présentatifs "c'est" / "il est" }\end{array}$ & $\begin{array}{l}\text { Expressions de temps avec "en", "il y } \\
\text { a", "depuis", "dans" }\end{array}$ \\
\hline Supports & & $\begin{array}{l}\text { Carte de France (accrochée } \\
\text { au tableau) } \\
\text {. Dossiers d'articles de presse } \\
\text { spécialisée décrivant des vins }\end{array}$ & $\begin{array}{l}\text {. Fascicule de l'exposition Byzance au } \\
\text { Grand Palais (octobre 2009-janvier } \\
\text { 2010) } \\
\text {. Atlas (carte du bassin méditerranéen) } \\
\text {. Texte de présentation de l'exposition } \\
\text { extrait du site internet du Grand Palais } \\
\text { - Dessin au tableau de la ligne } \\
\text { symbolisant le temps } \\
\text { Feuille d'exercice: phrases } \\
\text { comportant des marqueurs de temps, à } \\
\text { corriger si nécessaire }\end{array}$ \\
\hline
\end{tabular}

Les objets détaillés et les activités mises en œuvre dans les cours ALICE 1 et ALICE 2 
Tableau 3 - Aperçu du synopsis ${ }^{3}$ du cours ALICE 1.

Énumération de noms de vins français et de leurs régions de production, description de leurs caractéristiques

- Nommer des vins français et situer leur région de production

- Expliquer les conditions nécessaires pour produire du vin (chaleur, pluie) et faire émerger des éléments lexicaux liés au vin (raisin, vigne)

- Lister les éléments de lexique pour décrire le vin (en dominante, adjectifs)

Présentation orale de vins français sélectionnés

- Sélectionner un vin à présenter, à partir d'un ensemble de textes descriptifs de vins d'une région vinicole

- Présenter à l'oral un vin choisi, à partir d'un ensemble de textes descriptifs de vins d'une région vinicole

Dégustation d'un vin à l'aveugle

- Déguster un vin, le décrire et deviner ce que c'est

Tableau 4 - Aperçu du synopsis du cours ALICE 2.

Compréhension de la brochure et du texte de présentation de l'exposition

- Situer la thématique de l'exposition, à partir du visuel de la première page du fascicule de présentation de l'exposition

Repérage des grandes périodes historiques

- Situer les grandes époques historiques dans l'histoire de l'Occident (préhistoire, antiquité, moyen-âge, etc.) sur une ligne représentant le temps dessiné au tableau

Compréhension de la brochure et du texte de présentation de l'exposition

- Lister les dates clés de l'histoire de la ville d'Istanbul, à partir de la lecture des deux premières pages du texte de présentation de l'exposition De Byzance à Istanbul

- Décider de la véracité d'assertions par rapport au texte (vrai/faux)

Repérage des expressions de temps "en", "il y a", "depuis", "pendant", dans la brochure et réflexion sur leur sens et leur utilisation

- Repérer des exemples d'extraits du texte de présentation de l'exposition contenant les marqueurs de temps "en", "il y a", "depuis" et "dans"

- Conceptualiser la fonction des différents marqueurs de temps, à partir des exemples répertoriés au tableau en 4 colonnes, à partir du texte de présentation de l'exposition

- Vérifier l'utilisation juste des marqueurs de temps dans des phrases à corriger 


\section{Présentation et analyse des verbalisations d'Alice sur son agir}

\section{Analyse de contenus des commentaires d'Alice selon les trois pôles du triangle didactique}

L'analyse de contenus des verbalisations d'Alice a montré qu'un commentaire pouvait porter sur l'un des pôles du triangle didactique ou sur une combinaison de ces pôles. Ceci nous a amené à distinguer, quand cela était nécessaire, les différentes remarques contenues dans un même commentaire selon qu'elles concernaient Alice comme enseignante, les apprenants ou les objets de savoir traités. Par exemple, dans le premier commentaire qu'Alice fait à propos de ALICE 1, on trouve deux remarques, l'une à relier au pôle enseignant et plus particulièrement à la méthodologie de l'enseignement, l'autre à rapporter au pôle apprenant concernant les connaissances des apprenants :

Commentaire 1 (Code temps vidéo - CTV : 0'37")

ça c'est le brainstorming du début pour créer un p'tit peu de de dynamique [pôle enseignant/méthodes] euh + et de voir où en était où $\mathrm{y} \mathrm{z}$ en étaient dans ce point à la fois d'la culture française et de voir c'qu'i savaient de ce sujet-là [pôle apprenant/connaissances]

17 Ainsi, nous avons pu comptabiliser, pour le cours ALICE 1, 17 commentaires qui se répartissent en 25 remarques, et, pour le cours ALICE 2, 30 commentaires qui se répartissent en 45 remarques. Pour chaque cours, les remarques ont été rassemblées par thématique abordée et par pôle du triangle didactique concerné. Le tableau qui suit présente les différentes thématiques identifiées et comptabilisées dans chaque EAC:

Tableau 5 - Thématiques des remarques repérées dans les commentaires.

\begin{tabular}{|c|c|c|c|c|c|}
\hline & & $\begin{array}{l}30 \\
\text { rem }\end{array}$ & 1 & $\begin{array}{l}45 \\
\text { rem }\end{array}$ & $\begin{array}{l}2 \\
\text { ques }\end{array}$ \\
\hline \multirow{3}{*}{ Pôle enseignant } & Attitude et ressenti & 3 & \multirow{3}{*}{14} & 6 & \multirow{3}{*}{28} \\
\hline & Planification et gestion du temps & 4 & & 4 & \\
\hline & Savoirs d'ingénierie & $7^{4}$ & & 18 & \\
\hline \multirow{5}{*}{ Pôle apprenant } & Attitude & 2 & \multirow{5}{*}{9} & 4 & \multirow{5}{*}{7} \\
\hline & Centres d'intérêt & 1 & & & \\
\hline & Niveau de langue & 3 & & & \\
\hline & Capacités/connaissances & 12 & & 3 & \\
\hline & Difficultés & 3 & & & \\
\hline
\end{tabular}




\begin{tabular}{|l|l|l|l|l|l|}
\hline \multirow{4}{*}{ Pôle savoirs } & Objets abordés & & & 1 & \\
\cline { 2 - 3 } & Spécificités/difficultés & 2 & 2 & 5 & \multirow{2}{*}{10} \\
\cline { 2 - 3 } & Documents supports & & & 4 & \\
\cline { 2 - 3 } & & & & & \\
\cline { 2 - 3 }
\end{tabular}

18 Ce recensement macro fait apparaître que les verbalisations d'Alice sur ses cours concernent en dominante le pôle enseignant, et plus particulièrement les savoirs d'ingénierie et les questions d'ordre méthodologique (25 des 45 remarques repérées, dont 7 pour le cours ALICE 1 et 18 pour le cours ALICE 2).

19 Les tableaux suivants indiquent sur quels objets et activités des cours portent ces remarques à dimension méthodologique au sein des commentaires produits :

Tableau 6 - ALICE 1.

\begin{tabular}{|l|l|}
\hline Objets et activités & Commentaires \\
\hline Nommer des vins français et situer leur région de production & $\mathrm{n}^{\circ} 1$ \\
\hline Expliquer les conditions nécessaires pour produire du vin & \\
\hline Lister les éléments de lexique pour décrire le vin & $\mathrm{n}^{\circ} 6, \mathrm{n}^{\circ} 7$ \\
\hline $\begin{array}{l}\text { Sélectionner un vin à présenter, à partir d'un ensemble de textes descriptifs de } \\
\text { vins d'une région vinicole }\end{array}$ & $\mathrm{n}^{\circ} 10$ \\
\hline $\begin{array}{l}\text { Présenter à l'oral un vin choisi, à partir d'un ensemble de textes descriptifs de } \\
\text { vins d'une région vinicole }\end{array}$ & $\mathrm{n}^{\circ} 14, \mathrm{n}^{\circ} 15$ \\
\hline Déguster un vin, le décrire et deviner ce que c'est & $\mathrm{n}^{\circ} 16$ \\
\hline
\end{tabular}

Les commentaires d'Alice sur son premier cours se détaillent en remarques dont la teneur peut être résumée comme suit :

- dans le commentaire $n^{\circ} 1$, Alice pointe l'importance de l'activité remue-méninges du début de cours pour nourrir la dynamique de groupe ;

- dans les commentaires $n^{\circ} 6$ et $n^{\circ} 7$, elle souligne qu'expliquer le lexique en amont de la lecture des articles spécialisés sur le vin permet de gagner du temps pour la compréhension ;

- dans le commentaire $n^{\circ} 10$, elle exprime l'intérêt de donner un enjeu à l'activité de lecture et de préparation des présentations orales sur les vins choisis en proposant ensuite une dégustation à l'aveugle ;

- dans le commentaire $n^{\circ} 14$, elle explique la nécessité de noter au tableau les éléments d'information clés sur chaque vin pendant les exposés des étudiants (afin qu'ils gardent une trace du lexique et qu'ils puissent le réutiliser ensuite);

- dans les commentaires $n^{\circ} 15$ et $n^{\circ} 16$, elle questionne la manière et évoque l'intérêt de faire participer tous les apprenants. 
Tableau 7 - ALICE 2.

\begin{tabular}{|c|c|}
\hline Objets détaillés et activités mises en œuvre & Commentaires \\
\hline $\begin{array}{l}\text { Situer la thématique de l'exposition, à partir du visuel de la première page } \\
\text { du fascicule de présentation de l'exposition }\end{array}$ & $\mathrm{n}^{\circ} 3, \mathrm{n}^{\circ} 4, \mathrm{n}^{\circ} 5$ \\
\hline $\begin{array}{l}\text { Situer les grandes époques historiques dans l'histoire de l'Occident } \\
\text { (préhistoire, antiquité, moyen-âge, etc.) sur une ligne représentant le temps } \\
\text { dessiné au tableau }\end{array}$ & $\begin{array}{l}\mathrm{n}^{\circ} 6, \mathrm{n}^{\circ} 7, \mathrm{n}^{\circ} 8, \mathrm{n} \\
\circ 9\end{array}$ \\
\hline $\begin{array}{l}\text { Lister les dates clés de l'histoire de la ville d'Istanbul, à partir de la lecture } \\
\text { des deux premières pages du texte de présentation de l'exposition De } \\
\text { Byzance à Istanbul }\end{array}$ & $\begin{array}{ll}n^{\circ} 11, \quad n^{\circ} 12, \quad n \\
\circ 13\end{array}$ \\
\hline Décider de la véracité d'assertions par rapport au texte (vrai/faux) & $\begin{array}{l}n^{\circ} 15, \quad n^{\circ} 16, \quad n \\
\circ 18, n^{\circ} 19\end{array}$ \\
\hline $\begin{array}{l}\text { Repérer des exemples d'extraits du texte de présentation de l'exposition } \\
\text { contenant les marqueurs de temps "en", "il y a", "depuis" et "dans" }\end{array}$ & $\begin{array}{l}n^{\circ} 20, \quad n^{\circ} 22, \quad n \\
\circ 23\end{array}$ \\
\hline $\begin{array}{l}\text { Conceptualiser la fonction des différents marqueurs de temps, à partir des } \\
\text { exemples répertoriés au tableau en } 4 \text { colonnes, à partir du texte de } \\
\text { présentation de l'exposition }\end{array}$ & \\
\hline $\begin{array}{l}\text { Vérifier l'utilisation juste des marqueurs de temps dans des phrases à } \\
\text { corriger }\end{array}$ & $n^{\circ} 30$ \\
\hline
\end{tabular}

21 À nouveau, les commentaires d'Alice sur son deuxième cours se détaillent en remarques dont la teneur peut être résumée comme suit :

- dans les commentaires $n^{\circ} 3, n^{\circ} 4, n^{\circ} 5, n^{\circ} 6, n^{\circ} 13$ et $n^{\circ} 19$, Alice pointe l'importance des supports visuels utilisés et questionne le fait qu'elle $\mathrm{n}$ les a pas rendus assez visibles par les apprenants ;

- dans le commentaire $n^{\circ} 4$, elle ajoute une remarque concernant la dynamique de l'activité liée à l'utilisation adéquate du support visuel (une carte du bassin méditerranéen dans l'atlas montré aux apprenants) ;

- dans les commentaires $n^{\circ} 8$ et $n^{\circ} 9$, elle questionne la quantité d'informations qu'elle donne aux apprenants pour les aider à comprendre l'évolution d'une période historique à une autre (elle trouve que c'est à la fois peu et beaucoup et que ça ne guide pas vraiment la compréhension des apprenants) ;

- dans les commentaires $n^{\circ} 7, n^{\circ} 11, n^{\circ} 12, n^{\circ} 15, n^{\circ} 20, n^{\circ} 22$ et $n^{\circ} 23$, elle fait une série de remarques mettant en question ses manières d'animer les activités de compréhension et de repérage ;

- dans le commentaire $\mathrm{n}^{\circ} 16$, elle souligne que l'exercice de compréhension détaillée vrai/faux qu'elle avait fabriqué s'est avéré trop facile ;

- dans le commentaire $n^{\circ} 18$, elle poursuit son questionnement sur le support d'exercice vrai/ faux fabriqué en se demandant s'il s'agit en fait d'un exercice qui permet de développer la compréhension ou si c'est déjà de l'évaluation de la compréhension. 


\section{Indices de développement de l'agir enseignant d'Alice}

22 L'analyse micro des remarques permet d'identifier des indices du développement de l'agir d'Alice que l'on peut regrouper en trois catégories: les remarques qui confirment des pratiques effectives, les remarques qui les mettent en question, les remarques qui en envisagent d'autres afin de dépasser ce qu'Alice perçoit comme un blocage dans son agir.

\section{Confirmer des pratiques effectives}

Cette première catégorie d'indices du développement de l'agir enseignant d'Alice regroupe les remarques des commentaires $n^{\circ} 1, n^{\circ} 6, n^{\circ} 7, n^{\circ} 10, n^{\circ} 14, n^{\circ} 16$ du cours ALICE 1 et celles des commentaires $n^{\circ} 16$ et $n^{\circ} 22$ du cours ALICE 2 .

Dans ces remarques, Alice confirme cinq pratiques effectives qu'elle se voit utiliser lors du visionnement de ses cours :

- susciter une bonne dynamique au début d'un cours par exemple par le recours à une activité de type remue-méninges $\left(\mathrm{AL} 1 / \mathrm{n}^{\circ} 1\right)$;

- mener l'explication du lexique supposé nouveau et/ou difficile avant de faire lire et travailler des textes en compréhension (AL1/ $\mathrm{n}^{\circ} 6$ et 7$)$;

- donner un enjeu à une activité ou une tâche $\left(\mathrm{AL} 1 / \mathrm{n}^{\circ} 10\right)$;

- noter au tableau les éléments clés à retenir, à classer et éventuellement à réutiliser (AL1/n - $14 ;$ AL2 $/ n^{\circ} 22$ et 23$)$;

- le fait que tous les apprenants prennent part à une activité de production orale (AL1/n 16$)$.

Les remarques d'Alice sur ses pratiques effectives montrent que l'auto-confrontation l'amène à formuler des observations qui expriment et sans doute renforcent ses convictions méthodologiques sur les points listés.

Alice montre également à deux reprises qu'elle s'est approprié des termes spécifiques à la méthodologie de l'enseignement des langues désignant des phases possibles d'activités : elle désigne du terme "compréhension détaillée" l'activité menée à partir du questionnaire vrai/faux (AL2/n 16$)$; puis elle utilise les termes "repérage" et "conceptualisation" pour conclure sur l'activité qu'elle a voulu mettre en place avec les apprenants sur les expressions de temps (AL2/ $\mathrm{n}^{\circ} 22$ ). L'utilisation de ces termes spécifiques donne l'impression qu'Alice situe clairement la nature et la fonction des activités qu'elle commente, et leur évolution, de la planification à la mise en œuvre.

Le relevé de ces remarques de confirmation de pratiques effectives fait apparaître une partie du répertoire didactique d'Alice sur le plan méthodologique qui compose son style professionnel. Trois de ces remarques font écho aux options méthodologiques abordés en formation : le recours à une activité remue-méninges en début de cours, le fait de donner un enjeu à une tâche, la participation de tous les apprenants à une activité de production orale. Par contre, les deux autres renvoient à des manières de faire autres, voire opposées, aux savoirs d'ingénierie travaillés : l'explication par l'enseignant du lexique en amont de la lecture de textes, la notation au tableau par l'enseignant d'éléments clés présentés par des apprenants. 


\section{Mettre en question des pratiques effectives}

Cette deuxième catégorie d'indices du développement de l'agir enseignant d'Alice regroupe les remarques $d u$ commentaire $n^{\circ} 15$ du cours ALICE 1 et celles des commentaires $n^{\circ} 7, n^{\circ} 15$ et $n^{\circ} 18$ du cours ALICE 2 .

Dans ces remarques, Alice interroge quatre manières de faire qu'elle se voit utiliser :

- la mise en commun d'un travail fait en petits groupes par une personne seulement, ce qui amène Alice à se questionner sur les manières de faire participer tout le monde $\left(\mathrm{AL} 1 / \mathrm{n}^{\circ} 15\right)$;

- le fait que l'activité permettant de situer les grandes ères historiques se transforme en cours d'histoire où l'enseignante explique beaucoup d'éléments elle-même (AL2 $\left./ n^{\circ} 7\right)$;

- la trop grande durée de l'activité de compréhension du fascicule de l'exposition (AL2/n 15$)$ qui amène Alice à formuler le fait qu'elle aurait dû faire autrement pour mieux gérer le temps;

- la fonction réelle du questionnaire vrai/faux pour la compréhension détaillée du texte de présentation de l'histoire d'Istanbul (AL2/n $\left.\mathrm{n}^{\circ} 18\right)$. La façon dont se déroule l'activité telle qu'elle la visionne amène Alice à se demander si ce type même de questionnaire accompagne la compréhension ou plutôt l'évalue.

L'analyse de ces mises en question par Alice de son agir montre que l'auto-confrontation lui permet de pointer des zones de questionnement par rapport au développement de sa pratique enseignante. Ce qu'elle constate et commente est source pour elle $\mathrm{d}^{\prime}$ insatisfactions au niveau de la dynamique de groupe (AL1/ $\left.\mathrm{n}^{\circ} 15\right)$, de son rôle comme enseignante de langue $\left(\mathrm{AL} 2 / \mathrm{n}^{\circ} 7\right)$, de la gestion du temps (AL2/n $\left.\mathrm{n}^{\circ} 15\right)$, et de la visée réelle de l'activité (AL2 $\left./ \mathrm{n}^{\circ} 18\right)$. Ses questionnements sont en lien avec des principes ou des techniques travaillés en formation: mettre les apprenants en position de s'impliquer dans les activités, de découvrir les éléments d'informations par eux-mêmes, de rythmer les activités de manière à maintenir l'attention des apprenants, de vérifier la portée des activités entre apprentissage et évaluation. Ceci montre que les contenus abordés dans les savoirs d'ingénierie nourrissent la réflexion de l'enseignante novice placée face à ses actions situées.

\section{Faire évoluer ses pratiques effectives}

31 Cette troisième catégorie d'indices du développement de l'agir enseignant d'Alice regroupe les remarques des commentaires $n^{\circ} 4, n^{\circ} 11, n^{\circ} 12, n^{\circ} 13$ et $n^{\circ} 22$ du cours ALICE 2.

Dans ces remarques, Alice s'appuie sur l'interrogation de ses pratiques pour les faire évoluer vers d'autres possibilités de mise en œuvre. Ses remarques concernent :

- le recours à des supports visuels plus visibles pour favoriser une meilleure dynamique de l'activité (AL2 $/ \mathrm{n}^{\circ} 4$ et $\left.\mathrm{n}^{\circ} 13\right)$. Elle évoque par exemple la possibilité de projeter une grande carte du bassin méditerranéen plutôt que de montrer de loin une page d'atlas ;

- le choix d'autres types d'activités de compréhension détaillée pour gagner en temps, en dynamique et en efficacité de compréhension du document support (AL2/ $\mathrm{n}^{\circ} 11$ et $\mathrm{n}^{\circ} 12$ ). Alice envisage deux autres activités possibles pour faire repérer par les apprenants les dates clés de l'histoire d'Istanbul à partir de la lecture du fascicule de l'exposition : faire compléter un tableau qu'elle aurait préparé à l'avance, ou faire associer des étiquettes reprenant les dates et les événements clés de l'histoire de la ville. Ces deux idées représentent des alternatives à l'activité classique de questions/réponses qu'Alice se voit mettre en œuvre en 
visionnant le film de son cours tel qu'elle l'avait planifiée, mais cela lui semble long, laborieux et peut-être peu efficace par rapport à la compréhension du texte ;

- le fait de ne pas noter dans le tableau de repérage des expressions de temps des formes qui ne lui semblent pas correspondre à l'objet visé (AL2/n²2). Alice commente qu'elle devrait noter toutes les formes proposées par les apprenants y compris celles qui ne rentrent pas dans la catégorie d'expressions à relever, car le fait de lister toutes les propositions lui permettrait ensuite de solliciter un peu plus la réflexion des apprenants.

L'analyse de ces propositions d'autres pratiques par Alice concernant son propre agir montrent que l'auto-confrontation l'amène à entrevoir comment modifier la mise en œuvre des activités qu'elle planifie afin de les améliorer pour une meilleure appréhension de ces activités par les apprenants. À nouveau ici, ses interventions renvoient aux principes et techniques abordés en formation. Par exemple, les deux nouvelles techniques qu'elle envisage pour faire travailler la compréhension détaillée d'un document ont été explicitement travaillées. Ce constat pointe le fait que les savoirs d'ingénierie vus en formation ne sont pas automatiquement mis en œuvre par les enseignants novices, mais ils peuvent contribuer à développer leur réflexivité sur leur agir et les amener à le faire évoluer.

\section{Esquisse du portrait de l'agir enseignant en construction d'Alice}

Au terme de cette analyse partielle de l'agir d'Alice par le biais de ses commentaires sur ses pratiques effectives recueillis dans le cadre d'EAC, nous pouvons esquisser le portrait en construction de son style professionnel. Au fil de la mise en œuvre de ces deux cours et des commentaires qu'elle fait en les visionnant après coup, on perçoit que l'agir d'Alice est partagé entre deux conceptions du métier d'enseignant: l'enseignant qui fait et l'enseignant qui fait faire, ou encore, l'enseignant détenteur d'un savoir à transmettre et l'enseignant qui guide l'apprentissage du savoir par la mise en activité de l'apprenant. Alice se situe tantôt dans une posture de mise en activité des apprenants, tantôt dans la posture de l'enseignante qui "fait pour eux". Son style se construit entre confirmation et questionnement de ses pratiques effectives, avec pour propos de les faire évoluer vers une plus grande mise en activité des apprenants. Cette orientation de l'évolution de son agir se forge dans la dynamique réflexive dans laquelle Alice développe peu à peu ses pratiques effectives et se nourrit, entre autres, des ressources tirées de la formation. Ce mouvement est rendu visible par le biais du recours aux EAC.

\section{Conclusion : l'apport de l'auto-confrontation à l'analyse du développement de l'agir enseignant}

Comme nous l'avons rappelé en situant la notion d'agir enseignant, les actions de l'enseignant sont perçues d'abord au fil de l'analyse des interactions didactiques. Ce niveau d'analyse "en surface" permet un premier repérage des actions de l'enseignant, mais ne suffit pas pour en comprendre tous les ressorts. À travers l'analyse menée ici, le recours à l'auto-confrontation s'avère un outil précieux pour permettre au chercheur de saisir une partie du "dessous des cartes » des actions et des décisions de l'enseignant, ainsi que sa réflexivité sur le développement de son agir, qui émerge au fil des commentaires. L'auto-confrontation affine le grain d'analyse des interactions didactiques et permet de saisir au plus proche de l'action et au travers de la verbalisation la couleur 
didactique et méthodologique que l'enseignant donne à son action en situation et qui rend visible son style professionnel en construction.

\section{BIBLIOGRAPHIE}

Bigot, V. \& Cadet, L. (2011). "Comment la prise en compte des discours d'enseignants sur leurs pratiques renouvelle-t-elle l'analyse des interactions didactiques en classe de langue ?". In Bigot, V. \& Cadet, L. (dir.). Discours d'enseignants sur leur action en classe. Paris : Riveneuve éditions. pp. 11-29.

Borg, S. (2006). Teacher Cognition and Language Education : Research and Practice. London : Continuum.

Bronckart, J.-P. (1996). Activité langagière, textes et discours. Lausanne-Paris : Delachaux et Niestlé. Bronckart, J.-P. (2010). Une introduction aux théories de l'action. Genève : Université de Genève. Cambra Giné, M. (2003). L'approche ethnographique de la classe de langue. Paris : Didier. Causa, M. (2012). "Introduction - Le répertoire didactique : une notion complexe". In Causa, M. (dir.). Formation initiale et profils des enseignants de langues. Bruxelles : De Boeck. pp. 15-72.

Cicurel, F. (2002). "La classe de langue un lieu ordinaire, une interaction complexe". Aile, $\mathrm{n}^{\circ} 16$. pp. 146-163.

Cicurel, F. (2005). "Fiction, interaction, action : quelques jalons pour l'analyse des discours de la classe". In Mochet, M.-A. \& Barbot, M.-J. (dir.). Plurilinguisme et apprentissages : mélanges Daniel Coste. Lyon : ENS LSH. pp. 15-25.

Cicurel, F. (2007). "L'agir professoral, une routine ou une action à haut risque". In Plazaola Giger, I. \& Stroumza, K. (dir.). Paroles de praticiens et description de l'activité. Bruxelles : De Boeck. pp. 17-36.

Cicurel, F. (2011). Les interactions dans l'enseignement des langues - Agir professoral et pratiques de classe. Paris : Didier.

Cicurel, F. \& Bigot, V. (dir.). (2005). "Les interactions en classe de langue". Le français dans le monde, recherches et applications, numéro spécial juillet.

Cicurel, F. \& Rivière, V. (2008). "De l'interaction en classe à l'action revécue : le clair-obscur de l'action enseignante". In Filliettaz, L. \& Schubauer-Leoni, M.-L. (dir.). Processus interactionnels et situations éducatives. Bruxelles : De Boeck. pp. 255-273.

Clot, Y. (1999). La fonction psychologique du travail. Paris : PUF.

Clot, Y. \& Faïta, D. (2000). "Genres et styles en analyse du travail. Concepts et méthodes". Travailler, $\mathrm{n}^{\circ}$ 4. pp. 7-42.

Filliettaz, L. (2005). "Mise en discours de l'agir et formation des enseignants - Quelques réflexions issues des théories de l'action". Le français dans le monde, recherches et applications, numéro spécial juillet. pp. 20-31. 
Filliettaz, L., Schubauer-Leoni, M.-L. (2008). "Les processus interactionnels dans leurs dimensions interpersonnelles, socio-historiques et sémiotiques". In Filliettaz, L. \& Schubauer-Leoni, M.-L. (dir.). Processus interactionnels et situations éducatives. Bruxelles : De Boeck. pp. 7-39.

Garcia-Debanc, C. (2008). "De la configuration didactique au modèle disciplinaire en acte : trente ans de didactique du français avec Jean-François Halté". Pratiques, n 137/138. pp. 39-56.

Plazaola Giger, I. \& Stroumza, K. (dir.). (2007). Paroles de praticiens et description de l'activité. Bruxelles : De Boeck.

Rivière, V. (2005). "Aujourd'hui nous allons travailler sur... Quelques aspects praxéologiques des interactions didactiques". Le français dans le monde, Recherches et applications, numéro spécial juillet. pp. 96-104.

Rivière, V. \& Cadet, L. (2011). "Mise en perspective des savoirs professionnels dans des discours de retour d'expérience. Étude contrastée de deux enseignantes de français langue étrangère". Lidil, $\mathrm{n}^{\circ}$ 43. pp. 41-55.

Schneuwly, B. \& Dolz, J. (2009). Des objets enseignés en classe de français. Rennes : Presses universitaires de Rennes.

Tochon, F. V. (2000). "Recherche sur la pensée des enseignants : un paradigme à maturité". Revue française de pédagogie, $\mathrm{n}^{\circ} 133$. pp. 129-157.

\section{ANNEXES}

\section{Commentaires liés aux savoirs d'ingénierie}

\section{Corpus ALICE $1: 7$ remarques extraites de commentaires}

Commentaire 1 (CTV : 0'37')

ça c'est le brainstorming du début pour créer un p'tit peu de de dynamique euh + et de voir où en était où y $z$ en étaient dans ce point à la fois d'la culture française et de voir c'qu'i savaient de ce sujet-là+

Commentaire 6 (CTV : 11'23')

j'avais prévu une liste de d'adjectifs euh + pour justement parce que c'était l'objectif de ma séquence +

$\mathrm{Q}^{\circ}$ : La liste, vous l'aviez préparée à l'avance?

oh oui tout à fait oui oui + ça s'déroule comme j'avais pensé + ils répondent + quand même j'me suis rendu compte sur l'moment qu' c'était difficile de euh de d'expliquer certains termes euh euh + alors euh par exemple euh bon piquant c'est très simple euh tout le monde comprend + sensuel là aussi avec l'étymologie c'était facile mais y a un des autres mots euh un vin léger + un vin corsé un vin près du corps + qu'est-ce que ça peut vouloir dire pour quelqu'un ++

Commentaire 7 (CTV : 11'52')

c'était important qu'ils voient avant le vocabulaire pour gagner du temps + on avait qu'une heure + je voulais qu'ils comprennent les documents vite et qu'ils réutilisent ++

Commentaire 10 (CTV : 19'22") 
c'était une piquette que j'ai apportée (rit) + mais j'avais envie un peu d'leur euh d'leur euh qu'ily ait un axe qu'il y ait un p'tit peu un enjeu quoi le fait de lire et que ça débouche avec la pratique et visiblement ça leur a plu parce que c'était boire un p'tit coup euh ça marchait

Commentaire 14 (CTV : 28'33')

$\mathrm{Q}^{\circ}$ : Ça vous semblait important d'écrire pendant les présentations orales?

oh oui euh oui + j'ai fait un espèce de tableau avec euh le vin et puis les caractéristiques de chaque vin pour xxx les étudiants en fait + puisqu'i z étaient ensuite censés se servir de ça pour pouvoir qualifier aussi le vin quand ils le goûteraient +

Commentaire 15 (CTV : 32'18')

j'm'interroge sur le fait de faire une restitution par une personne quand y a trois personnes qui travaillent ensemble bon euh + forcément celui qui se dévoue ou qui a envie de parler aux autres travaillera travaille plus que les autres et + et finalement la vraie question c'est comment faire pour que tout le monde participe vraiment tout le monde travaille vraiment dans dans l'travail de groupe + euh et puis ça c'est une question + mais c'est une manière de faire et la deuxième manière bon euh à chaque fois j'ai dit qui vient et $y$ a toujours quelqu'un qui vient

Commentaire 16 (CTV : 45'59')

j'voulais qu'tout le monde participe + donc c'est vrai qu'ça donne une impression de brouillon mais en gros euh + j'étais un peu à l'affût de savoir de d'avoir la moindre euh + expression des uns ou des autres ouais

\section{Corpus ALICE 2 : 9 des 18 remarques extraites de commentaires}

Commentaire 4 (CTV : 3'21')

effectivement j'me dis que i faudrait qu'j'utilise plus euh euh + enfin plus de matériel pour avoir une grande carte projetée grand + là j'suis trop encore dans des papiers dans des + une façon d'travailler qui est qui est + un peu + oui pas assez dynamique p'têtre pour ça pour ce côté-là + non écrire au tableau si ça ça va + mais l'atlas non c'était pas une bonne idée

Commentaire 7 (CTV : 5'34')

ça les a intéressés + mais c'était un cours d'histoire + là j'suis en train de si de situer la préhistoire + $c^{\prime}$ est-à-dire j'les sollicite et $i$ savent certaines choses + et j'complète mais +

Commentaire 11 (CTV : 16'40")

euh + j'me dis qu'c'aurait été plus efficace en termes de + et surtout plus + j'aurais gagné du temps en faisant en préparant un + un tableau ou un + au lieu de faire à l'oral cette euh + ce questionnement euh + j'aurais dû préparer un tableau j'pense + une fois qu'i avaient lu + euh leur leur donner quelque un travail à faire par écrit ou + làj'trouve qu'c'est long + ça m'paraissait très long

Commentaire 12 (CTV : 16'54")

j'aurais pu + mettre des dates + mettre des événements tout mélanger et puis leur demander de retrouver + par exemple + ça aurait été trop compliqué p't'être + mais là tout à coup j'me dis c'est intéressant cette idée

Commentaire 13 (CTV : 17'52") 
c'est c'que j'disais tout à l'heure hein ça ça va pas du tout + j'ai beau être près d'eux parce que la salle est petite ben $i$ voient pas $i$ peuvent pas voir donc là il fallait utiliser le rétroprojecteur ouais ouais

Commentaire 15 (CTV : 28'10')

ben la compréhension elle aurait dû prendre beaucoup moins de temps et j'aurais dû m'y prendre autrement quoi

Commentaire 16 (CTV : 31'09")

là c'est la compréhension détaillée et donc c'est un questionnaire vrai faux voilà + j'avais essayé d'reformuler un peu les questions euh + y avait des choses très faciles du style euh est ce que tel évé + tel événement s'est passé à telle date et en fait dans l'texte on voyait qu'ça s'était passé à une autre date donc fallait trouver bon + et puis y avait d'autres questions plus difficiles alors là j'ai pas entendu j'me souviens plus quelle était la question difficile

$\mathrm{Q}^{\circ}$ : Vous pensiez qu'ce s'rait difficile...

oui et puis ils savaient tous ils avaient comp soit ils le savaient par leur culture soit ils l'ont tout d'suite trouvé dans l'texte là ++ et là j'ai peur de r'partir dans l'histoire avec les croisades

Commentaire 18 (CTV : 33'54')

bon en voyant le le + la correction du vrai faux je m'interroge euh + sur euh le fait que ce questionnaire 'fin ce + ce questionnaire euh + est ce qu'il permet de faire avancer la compréhension + ou est ce qu'il euh permet d'évaluer la compréhension et + 'fin là visiblement + euh mmh + y a des choses qui n'ont pas été comprises + mais le fait de l'faire à l'oral comme ça et de corriger permet de reprendre des points et de du coup de mieux comprendre le texte + mais quand même souvent j'me pose la question 'fin j'suis pas très satisfaite de euh de ce genre de questionnaire parce que j'me dis ben ceux qui n'ont pas compris ils peuvent pas le faire en réalité +

Commentaire 22 (CTV : 42'39')

non y a quelque chose qui va pas + parce que il me l'a proposé (la proposition « en parallèle ») j'aurais dû l'marquer j'aurais dû l'marquer + et ensuite revenir en disant est ce que ça va bien dans cette colonne + ben i'm semble

$\mathrm{Q}^{\circ}$ : Vous avez organisé un classement au tableau pour lancer les étudiants dans le classement de ces marqueurs, au fur et à mesure de ce que disent les étudiants, vous complétez les colonnes, et après vous voulez en faire quoi ?

ben j'veux leur dire euhj'veux qu'ils euh + trouvent par eux-mêmes pourquoi dans certains cas on dit en pourquoi on dit il y a et qu'i et qu'ils arrivent à sentir l'idée de la durée de la continuité la date + c'était ça mon idée + c'est du repérage et d'la conceptualisation

\section{NOTES}

1. Alice entreprenait alors un master en didactique des langues en vue d'une reconversion professionnelle dans le domaine de l'enseignement du FLE/S en France.

2. Les étudiants chinois étaient inscrits dans un programme de mise à niveau en français (dans le cadre d'un "Diplôme universitaire de français de spécialité"). Les objectifs du cours consistaient à amener les étudiants chinois à développer leurs compétences de communication orale et écrite 
en français au niveau indépendant B2 afin qu'ils puissent poursuivre leur cursus de formation universitaire à l'université Paris Descartes, dans la filière scientifique de leur choix.

3. La technique du synopsis a été utilisée pour donner une vision globale, synthétique et hiérarchisée des objets langagiers abordés en lien avec les activités d'enseignement/ apprentissage mises en œuvre dans les cours animés par Alice (cf. Schneuwly \& Dolz, 2009 : 89-100). Les synopsis ont été construits à partir de l'analyse des transcriptions des cours.

4. Les commentaires comportant des remarques portant sur les savoirs d'ingénierie sont reproduits en annexe (les 7 commentaires d'ALICE 1, et 9 des 18 commentaires d'ALICE 2).

\section{RÉSUMÉS}

Cet article décrit un exemple de recours à l'utilisation de la technique de l'auto-confrontation simple dans le cadre d'une recherche portant sur le lien entre la formation à la méthodologie de l'enseignement du FLE et le développement de l'agir d'enseignants novices. Après avoir situé la notion d'agir enseignant et la méthodologie de recherche utilisée avec la technique d'autoconfrontation, l'article propose l'esquisse du portrait de l'agir en développement d'une enseignante novice grâce à l'analyse des commentaires de cette enseignante produits dans le cadre d'entretiens d'auto-confrontation réalisés à la suite de deux de ses premières prises en main de cours de FLE. Cette analyse illustre en quoi le recours à l'auto-confrontation permet de cerner le développement de l'agir d'enseignants novices.

This article describes the use of stimulated recall in a research focused on the link between French second language teachers' training (with a focus on teaching skills) and the development of beginning teachers' actions. First of all, the article presents the notion of teachers' actions and the research methodology used with the stimulated recall technique. The article then sketches out the development of a beginning teacher's actions through the comments that this teacher has produced in stimulated recall interviews, organized after two of her first experiences of teaching French as a foreign language. This analysis shows how the use of stimulated recall makes it possible to access the development of beginning teachers' actions.

\section{INDEX}

Mots-clés : auto-confrontation, agir enseignant, savoirs d'ingénierie, style professionnel Keywords : stimulated recall, teachers' actions, teaching skills, professional style

\section{AUTEUR}

\section{VÉRONIQUE LAURENS}

Université Sorbonne Nouvelle - Paris 3, France

Véronique Laurens est maître de conférences en didactique du FLE à l'université Sorbonne Nouvelle - Paris 3 (laboratoire Diltec EA 2288). Ses intérêts de recherche concernent la 
construction de l'agir enseignant de FLE, sur le plan de la méthodologie (objets et démarches de formation en FLE), à travers l'analyse des interactions didactiques et des auto-commentaires (observation de l'émergence de l'action méthodologique enseignante dans les discours de la classe et dans les entretiens d'auto-confrontation).

Toile : www.univ-paris3.fr/laurens-veronique-289367.kjsp?RH=1178827308773

Courriel : veronique.laurens[at]univ-paris3.fr 